\title{
A INFLUÊNCIA DA IRRADIAÇÃO SOLAR NOS HARMÔNICOS GERADOS POR SISTEMAS SOLARES ON-GRID E SEU EFEITO NO FATOR K DO TRANSFORMADOR MONOFÁSICO
}

\author{
Mateus C. Machado - mateusmachadoeng@gmail.com \\ CEFET/RJ - Campus Angra dos Reis \\ Rua do Areal, 522, Parque Mambucaba \\ 23953-030 - Angra dos Reis - RJ
}

Emmanuel L. C. Saldanha - emmanusal@gmail.com

Cintia F. F. Carraro - cintia.carraro@cefet-rj.br

Resumo: Com o aumento da competitividade no mercado de trabalho, os profissionais necessitam acumular cada vez mais habilidades e conhecimento para obterem destaque em suas carreiras. Deste modo, adotar um método de ensino que desenvolva habilidades $e$ construa conhecimento amplo é desafiador. A simulação computacional é uma habilidade do futuro e combinada com a observação de problemas de engenharia e a multidisciplinaridade de projetos, pode ser um bom método de ensino. Assim, neste trabalho fez-se todo o projeto de um sistema fotovoltaico conectado à rede elétrica e buscou-se avaliar através de simulação computacional o efeito dos harmônicos gerados pelo sistema em um transformador. Como resultado, constatou-se que os níveis de irradiação alteram os níveis dos harmônicos gerados pelo sistema, podendo causar sérios problemas à rede e ao transformador de conexão à rede por alterar o fator $K$ do mesmo. Esta metodologia de ensino que alia recursos computacionais com a multidisciplinaridade envolvida em projetos de engenharia e a identificação de tendências do sistema elétrico se mostra um modelo interessante pois não só permite o desenvolvimento de habilidades como também o conhecimento amplo e o olhar crítico do engenheiro.

Palavras-chave: Sistema fotovoltaico. Inversor on-grid. Fator K. Transformador. Desafio na engenharia.

\section{INTRODUÇÃO}

O mercado de trabalho está cada vez mais competitivo e nas áreas de engenharia isto não é diferente. Logo, para o profissional obter destaque em sua carreira, são necessárias habilidades que o diferencie dos demais.

Neste aspecto, a simulação computacional é uma habilidade de alto valor, pois permite, por exemplo, conhecer o funcionamento e analisar um projeto antes de sua implementação, fornecendo dados que podem contribuir para o seu aprimoramento.

Ter conhecimento em áreas diversas da engenharia e sobre como elas interagem entre si também é importante na elaboração de um projeto ou estudo. Esta multidisciplinaridade dá ao 
engenheiro a capacidade de enxergar os problemas de modo amplo, o que influencia positivamente sua tomada de decisão e melhora a qualidade do projeto.

Outra habilidade do engenheiro do futuro é saber enxergar desafios futuros, observando tendências do mercado energético.

O desenvolvimento de artigos científicos supervisionado por professores pode ser uma opção barata e motivadora de ensino que supera dificuldades atuais, como o impedimento de aulas presenciais, pois com o acesso fácil a outros artigos e simuladores computacionais, o aprendizado de novos temas pode ser realizado mesmo em meio à crise educacional provocada pela pandemia do Covid-19. Promovendo um ensino baseado nas metodologias ativas de aprendizagem, onde o aluno deixa de ser um mero espectador e passa a fazer parte do processo, construindo soluções e buscando a interdisciplinaridade dos temas. Além disso, este método pode ser utilizado também em ensino presencial.

Desta forma, este artigo aborda um novo método de ensino, incentivando os alunos a identificarem problemas de engenharia e suas possíveis soluções, assim realizando estudos de caso com o auxílio de simulações computacionais e todo o processo sendo supervisionado pelo professor.

Foi escolhido o caso de um sistema fotovoltaico de proporção residencial conectado à um transformador de distribuição. Através de diversas simulações foi analisado como o sistema fotovoltaico afeta o Fator $\mathrm{K}$ do transformador e a mudança de harmônicos gerados com a variação da irradiação, tendo em vista que alterações bruscas desses parâmetros num transformador que não foi dimensionado para suportar essas mudanças podem causar sérios problemas de sobreaquecimento e qualidade de energia que podem causar sérios problemas de sobreaquecimento e qualidade de energia que podem afetar outras cargas conectadas ao mesmo e comprometer o tempo de vida útil.

\section{MÉTODO DE ESTUDO}

O estudo do fator K causado por distorções harmônicas é feito com o auxílio de simulação computacional e para simular é necessário desenvolver e dimensionar toda a instalação, desde o painel fotovoltaico à rede elétrica.

Nos próximos subtópicos são apresentados os componentes dimensionados.

\subsection{O sistema de geração fotovoltaico conectado à rede}

O sistema de geração fotovoltaico utilizado possui dois estágios de operação, os quais correspondem à conversão CC-CC e inversor (ROZENBLAT, 2017).

A Figura 1 demonstra um sistema fotovoltaico de dois estágios clássico, conectado à rede.

Figura 1- Sistema fotovoltaico conectado à rede

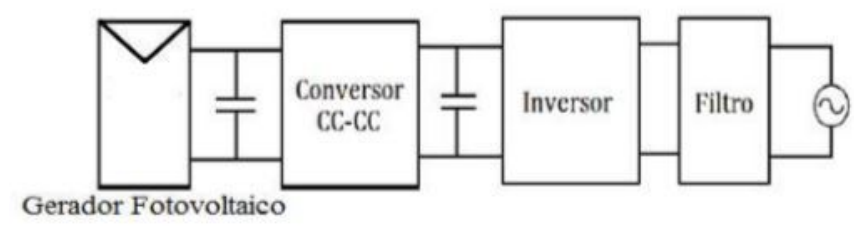

Fonte: Sousa, 2016. 
A Figura 1 também apresenta um filtro após o inversor, o qual serve para atenuar frequências indesejadas no sinal de saída geradas pelo chaveamento do inversor.

Este sistema necessita de controle para sincronizar a saída do inversor à frequência da rede, garantir bom fator de potência e garantir a máxima geração do sistema fotovoltaico.

Neste trabalho, adicionou-se à topologia da Figura 1 um transformador abaixador de tensão entre a rede e o sistema fotovoltaico. Considerou-se uma rede elétrica monofásica de $13.8 \mathrm{kV}$ e frequência $60 \mathrm{~Hz}$ e o transformador abaixador reduz tal tensão para $127 \mathrm{~V}$ no lado do sistema fotovoltaico.

\subsection{Arranjo fotovoltaico}

Para dimensionar o arranjo fotovoltaico é necessário primeiro saber a demanda elétrica residencial. Segundo a EPE em 2019, em 2018 o consumo médio residencial no subsistema elétrico que atende às regiões Sudeste e Centro-Oeste do país foi de $172,8 \mathrm{kWh} / \mathrm{mês}$, o que corresponde a $5,76 \mathrm{kWh} /$ dia.

Considerando que a demanda média diária é $5,76 \mathrm{kWh} /$ dia e que o tempo de insolação médio anual predominante para a região Sudeste/Centro-Oeste, o qual é de 06 horas diárias (TIBA, et al; 2000), determina-se que o sistema deve ser capaz de gerar no mínimo 960 Watts de potência por hora de geração.

Para tal, considerou-se um arranjo com duas strings, cada string com 3 placas solares do modelo CS6P-265P de $265 \mathrm{~W}$ de potência, da fabricante Canadian Solar. Este modelo de placa foi escolhido por já ter sido avaliada em trabalhos anteriores (SALDANHA, et al; 2019) e ter apresentado bons resultados.

Este sistema apresenta geração de aproximadamente $1590 \mathrm{~W}$ no ponto de potência máxima, podendo gerar um pouco mais de $1 / 3$ do que seria solicitado do sistema. Esta configuração possui um bom fator de dimensionamento $(0,63)$ e apresenta pouca perda de energia ao considerar um ano de geração, segundo exposto por Pereira e Gonçalves em 2008.

Para simular o painel fotovoltaico foi usado o modelo de código proposto por Casaro e Martins, 2008. Se trata de um algoritmo que usa como dados de entrada a tensão do arranjo, a irradiação que atinge os geradores, em $\mathrm{W} / \mathrm{m}^{2}$, e a temperatura ambiente. Aplicando as equações para células fotovoltaicas, o código fornece como saída a corrente do arranjo fotovoltaico.

\subsection{Conversor $\mathrm{CC}-\mathrm{CC}$}

Os conversores CC-CC são formados por elementos passivos associados a semicondutores chaveados e tem como objetivo controlar o fluxo de energia de uma fonte para outra (MARTINS, BARBI; 2006).

Neste trabalho foi usado um conversor boost clássico e seu dimensionamento foi feito de acordo com os passos seguidos por Silva em 2015. Os valores calculados para o indutor, capacitor e duty cycle do conversor foram, respectivamente: $207,345 \mu \mathrm{H} ; 17,31 \mathrm{mF}$ e 0,489 . Assim, o conversor eleva a tensão gerada de $91,8 \mathrm{~V}$ no ponto de máxima potência para 179,61 $\mathrm{V}$, tensão de pico da rede elétrica monofásica adotada.

Ainda segundo Silva, foi calculado um capacitor de entrada para o sistema do boost, com o objetivo de minimizar a oscilação inicial na geração. $O$ valor de capacitância encontrado foi $369,04 \mu \mathrm{F}$.

O chaveamento do conversor é feito por PWM (pulse width modulation) à uma frequência de $25 \mathrm{kHz}$ e nele está ligado o sistema de rastreamento de máxima potência. 


\subsection{MPPT}

Conectado ao conversor está o MPPT (rastreador do ponto de máxima potência), o qual rastreia o ponto de máxima potência do arranjo e garante que o sistema opere sempre em sua potência máxima.

Há diferentes modelos de MPPT e Pereira, em 2012, fez um apanhado e comparou métodos clássicos de rastreamento do ponto de máxima potência. $\mathrm{O}$ resultado é que o método de condutância incremental apresenta boa resposta e converge rapidamente. Assim, o método de condutância incremental foi escolhido para este trabalho.

A Figura 2 apresenta o fluxograma do método da condutância incremental.

Figura 2 - Fluxograma do Método da Condutância Incremental

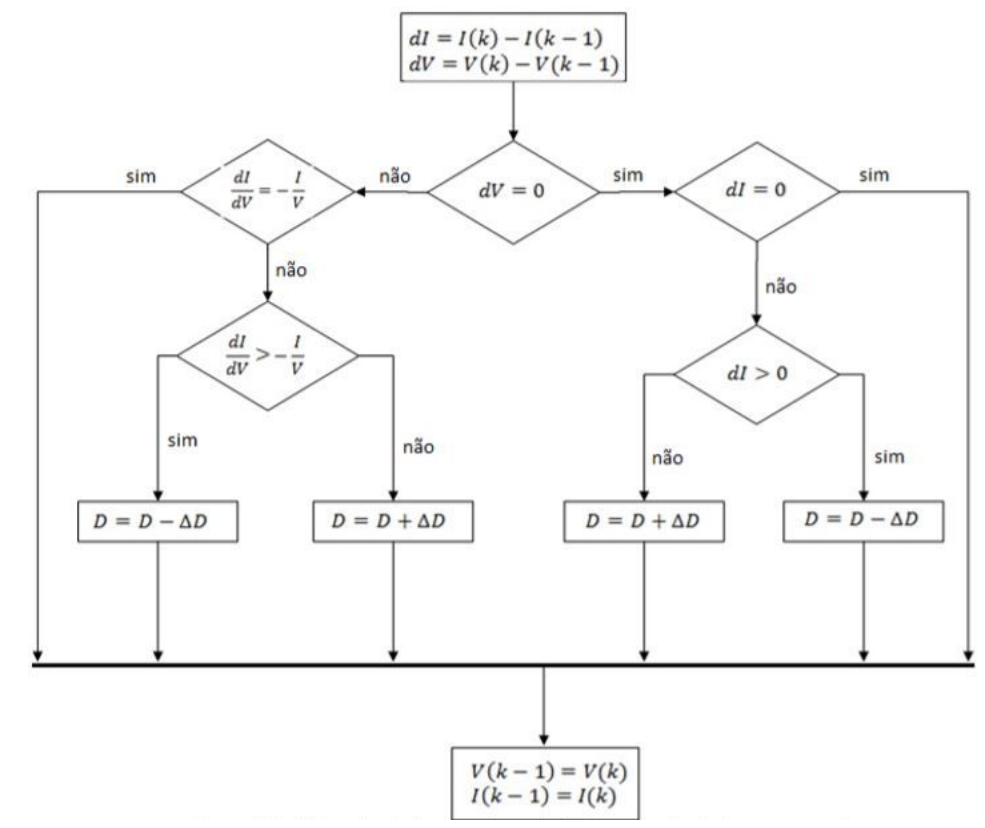

Fonte: Pereira, 2012.

Pelo fluxograma da Figura 2, nota-se que o método analisa as derivadas dos sinais de corrente e tensão gerados pela placa e realiza correções no duty cycle do conversor. Se as derivadas forem nulas, o duty cycle é mantido e consequentemente os sinais de saída. Se não, deve-se aumentar ou diminuir o duty cycle a fim de controlar a tensão de saída do arranjo fotovoltaico.

\subsection{Inversor}

O inversor é o componente fundamental de uma usina solar conectada à rede (MOJUMDAR et al; 2011), já que seu papel é transformar o sinal CC em CA e nele é feito o controle que sincroniza o sinal produzido com o sinal da rede.

Neste trabalho optou-se pelo modelo ponte completa, que é composto de 4 MOSFETs que funcionam como chaves, um par para o ciclo positivo e um par para o ciclo negativo.

O sistema de controle do inversor também possui variações, como Sousa revisou em 2016. O modelo adotado neste trabalho foi o proposto por Freitas em 2013 e adotado por Sousa. Este controle é feito por dois controladores PI e exposto na Figura 3. 


\section{Evento On-line}

Figura 3 - Sistema de controle do inversor

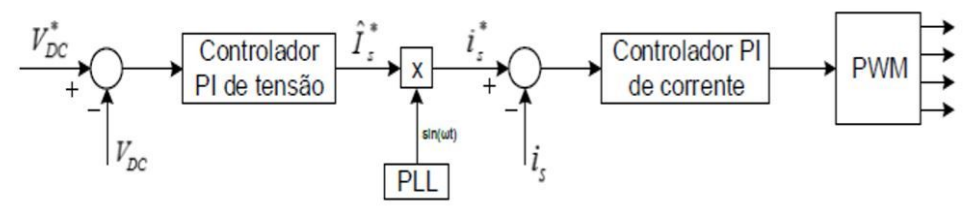

Fonte: Freitas, 2013.

Este sistema de controle busca manter constante a tensão no barramento CC ao comparar a tensão atual com uma tensão de referência e corrigir o erro por meio de um controlador PI. O sinal corrigido é transformado em uma senoide em fase com a rede ao ser multiplicado pelo sinal do PLL (phase locked loop). Este sinal é comparado com a corrente atual do sistema e o erro corrigido por outro controlador PI, cuja saída é base para o controle PWM do inversor.

$\mathrm{O}$ inversor tem como característica a geração de harmônicos nos sinais de saída, principalmente harmônicos ímpares. Isto ocorre pelo sinal de saída ser uma onda retangular periódica devido ao chaveamento, o qual foi adotado como $10 \mathrm{kHz}$, dos semicondutores que compõem o inversor.

Assim, foi dimensionado um filtro L, como feito por Silva em 2015, para reduzir os harmônicos e acoplar o sistema fotovoltaico à rede. A indutância encontrada para o filtro foi de $5,84 \mathrm{mH}$.

Para a carga a ser alimentada pelo inversor, considerou-se o mesmo consumo residencial médio adotado no dimensionamento do arranjo fotovoltaico, mas com um fator de potência de 0.92 indutivo. Através de cálculos, chegou-se a um valor de resistência de $56.8816 \Omega \mathrm{e}$ indutância de $150.8831 \mathrm{mH}$.

\subsection{Fator K}

O Fator K quantifica os efeitos térmicos provocados por correntes harmônicas, que são acumulativos. De forma mais técnica, o Fator $\mathrm{K}$ é o efeito total, em p.u., produzido no transformador pela corrente distorcida acima do efeito produzido pela corrente senoidal nominal (PAGUANINI, JANUÁRIO; 2014).

A partir deste parâmetro se faz cálculos para sobre dimensionamento do transformador, em vista de normalizar a vida útil do equipamento.

Para calcular o Fator K de forma autônoma em cada simulação, foi desenvolvido um código implementando a equação 1, fornecida por Teixeira em 2009.

$$
\text { Fator } K=\sum_{h=1}^{11} \frac{\left(I_{h} \times h\right)^{2}}{I_{1}^{2}}
$$

Onde: h: é a ordem do harmônico

$\mathrm{I}_{\mathrm{h}}$ : é a corrente correspondente à frequência harmônica

\section{RESULTADOS}

Para analisar como a irradiação solar afeta a geração de harmônicos e, por sua vez, afeta o Fator K do transformador, foram feitas simulações variando a irradiação num passo de 1000 $\mathrm{W} / \mathrm{m}^{2}$ em cada uma. Foram considerados 150 ciclos do espectro de corrente em cada simulação para extrair os valores das componentes harmônicas. 


\section{COBENCE e III Simpósio Internacional 2020 de Educação em Engenharia da ABENGE}

Para interpretar os valores do Fator K é preciso entender que ele é dado em p.u., e a referência é a corrente da frequência fundamental, ou seja, os valores serão sempre maiores que 1, pois o Fator $\mathrm{K}$ unitário é a representação dos efeitos térmicos provocada pela corrente fundamental.

Os valores de corrente foram aferidos na saída do transformador, tanto do lado de baixa quanto do lado de alta.

A Figura 4 apresenta a variação do Fator K segundo a irradiação solar.

Figura 4 - Fator K em função da Irradiação

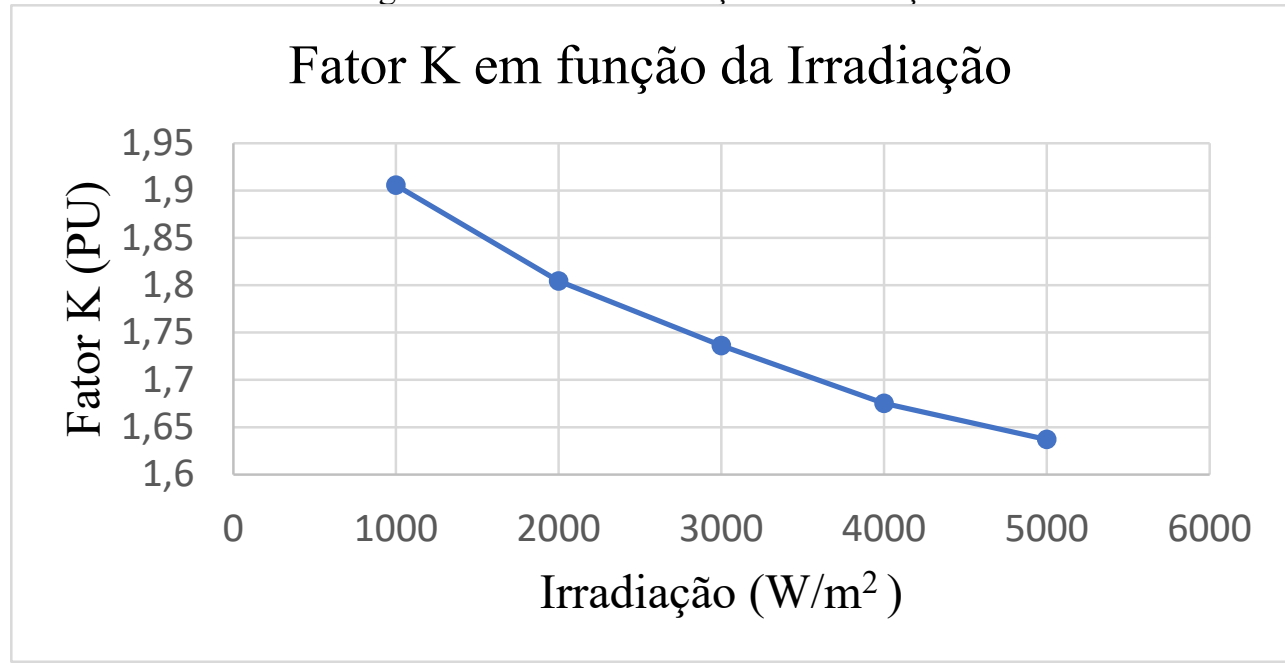

Fonte: Autoria própria.

Neste gráfico observa-se que com o aumento da irradiação há uma diminuição do Fator K, ou seja, quanto maior a irradiação, menor é a potência transformada em energia térmica no transformador, provocada pela influência de distorções harmônicas.

Isto indica um possível encarecimento do projeto devido ao uso de filtros mais robustos, em lugares com baixa irradiação, como o sul do país.

Para entender a queda do Fator K, foi analisada o THD da corrente (THDi), o qual é apresentado na Figura 5.

Figura 5 - THD de corrente em função da Irradiação

\section{THDi em funçâo do Irradiação}

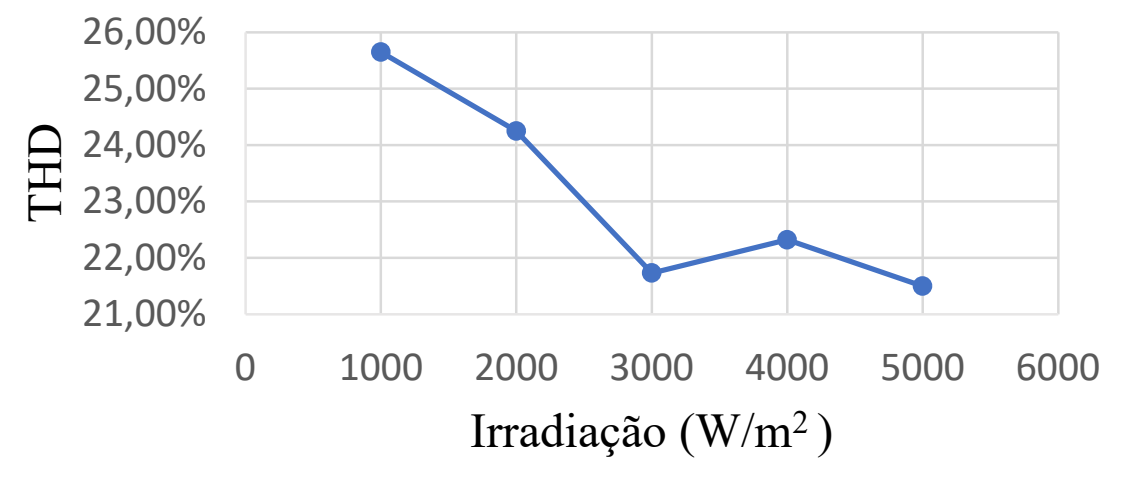


Fonte: Autoria própria.

Com isso podemos ver que o THD da corrente também decresce com o aumento da irradiação. Isto ocorre porque com o aumento da irradiação, aumenta a geração da onda de frequência fundamental, fazendo com que as outras frequências tenham uma influência menor.

O Quadro 1 mostra os harmônicos individualmente.

Quadro 1 - Harmônicos de corrente na rede

\begin{tabular}{|c|c|c|c|c|c|c|c|}
\hline \multirow{2}{*}{ Irradiação } & \multirow{2}{*}{$\begin{array}{c}\text { THDi } \\
(\%)\end{array}$} & \multirow{2}{*}{$\begin{array}{l}\mathrm{N}^{\circ} \mathrm{de} \\
\text { ciclos }\end{array}$} & \multicolumn{5}{|c|}{ Harmônicos (\%) } \\
\hline & & & ordem 3 & ordem 5 & ordem 7 & ordem 9 & ordem 11 \\
\hline 1000 & 25,65 & 150 & 23,54 & 8,45 & 4,20 & 2,60 & 1,74 \\
\hline 2000 & 24,25 & 150 & 22,26 & 7,96 & 4,07 & 2,45 & 1,64 \\
\hline 3000 & 21,73 & 150 & 19,95 & 7,12 & 3,65 & 2,33 & 1,56 \\
\hline 4000 & 22,32 & 150 & 20,51 & 7,30 & 3,74 & 2,25 & 1,51 \\
\hline 5000 & 21,50 & 150 & 19,75 & 7,02 & 3,60 & 2,17 & 1,45 \\
\hline
\end{tabular}

Fonte: Autoria própria.

Pelo Quadro 1, nota-se que o harmônico de maior influência em todas as irradiações é o de $3^{\mathrm{a}}$ ordem e como este tem uma importância maior quando se tratando da rede elétrica e de transformadores, torna-se necessário uma análise mais detalhada.

Assim, a Figura 6 apresenta o nível de harmônicos de $3^{\text {a }}$ ordem de acordo com a irradiação.

Figura $6-3^{\circ}$ Harmônico de corrente em função da Irradiação

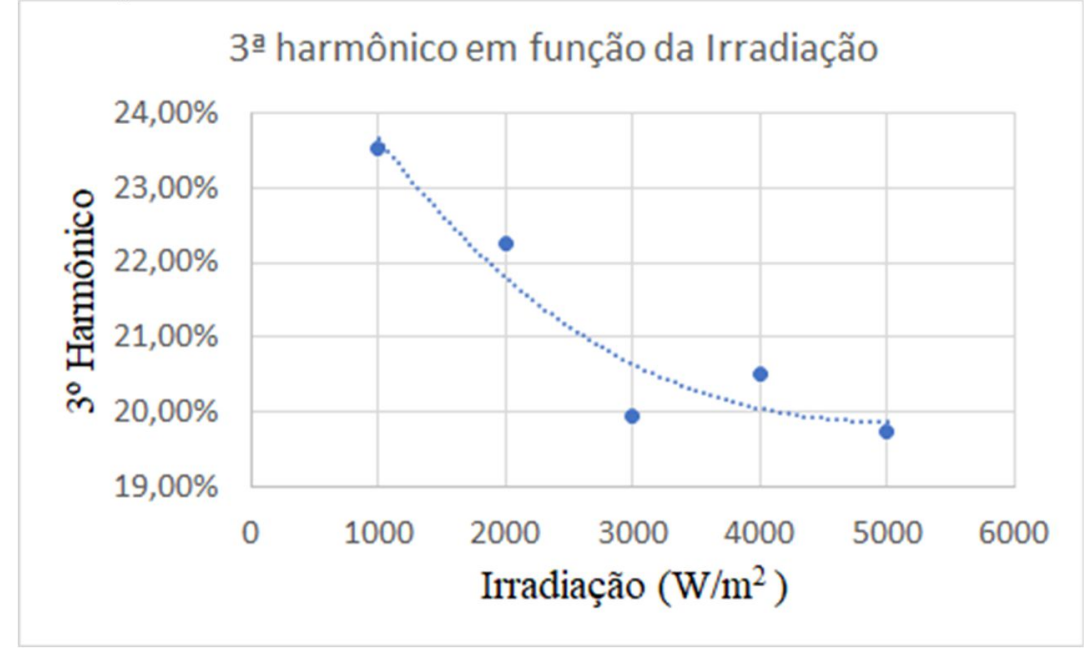

Fonte: Autoria própria.

Como mostra o gráfico da Figura 6, com a diminuição da irradiação, pode-se notar um incremento do harmônico de terceira ordem, isto pode ser um problema considerando vários sistemas fotovoltaicos conectados no mesmo transformador trifásico, pois os harmônicos de 3 ordem entram em fase, causando um aumento na amplitude do mesmo e podendo superar a fundamental, resultando em sérios problemas na rede e no equipamento (CHAPMAN, 2013).

Para analisar a influência direta do harmônico de $3^{\mathrm{a}}$ ordem no transformador, a Figura 7 apresenta o Fator K em função do $3^{\circ}$ harmônico. 
Figura 7 - Fator K em função do $3^{\circ}$ Harmônico

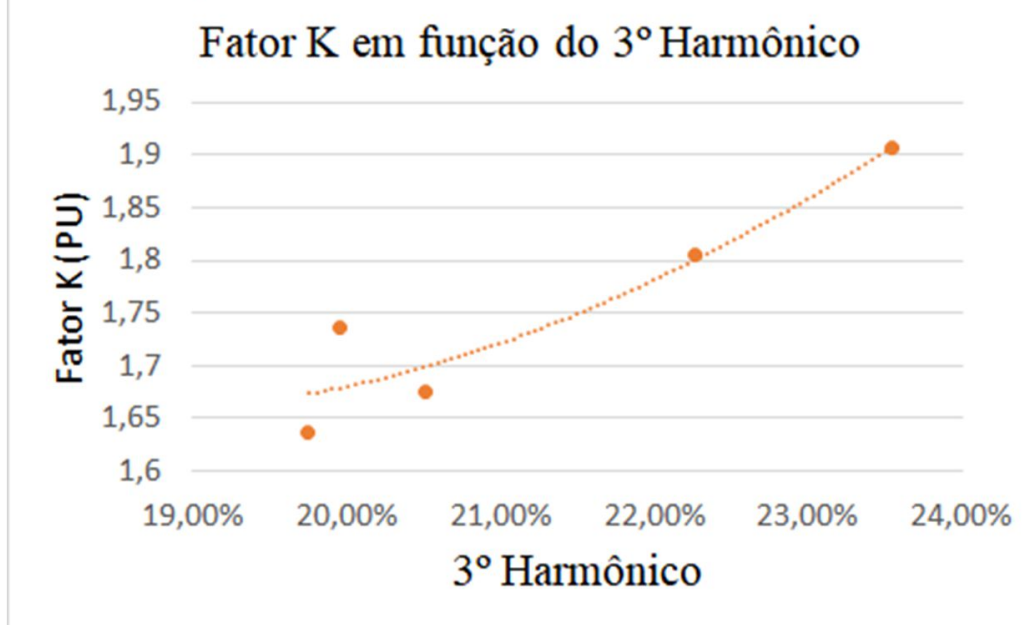

Fonte: Autoria própria.

Como mostra o gráfico da Figura 7, mesmo se tratando de transformadores monofásicos, este harmônico é o que mais influencia o Fator K. Ou seja, se tratando de um transformador trifásico, esta curva seria muito mais íngreme, acarretando num aumento de temperatura súbito e podendo provocar sérios danos ao transformador.

Deste modo, se faz necessário um filtro para esta frequência em redes de geração distribuída de sistemas fotovoltaicos para evitar a redução da vida útil dos transformadores.

\section{CONSIDERAÇÕES FINAIS}

Foi comprovado que o nível de irradiação altera os níveis de distorção harmônica de corrente e influencia o harmônico de terceira ordem, consequentemente alterando o Fator K do transformador e sua perda de potência, assim podendo causar sérios problemas tanto ao transformador quanto à rede. Logo, é interessante adicionar o uso de um filtro de harmônico de terceira ordem.

Portanto, o estudo da irradiação em projetos fotovoltaicos não é necessário apenas para determinar a potência do mesmo, mas para avaliar se transformador que estará conectado será muito prejudicado e com isso evitar prejuízos futuros.

Com o desenvolvimento das simulações e análises críticas. o método de ensino teve o efeito desejado, onde os alunos aprenderam sobre o funcionamento de sistemas fotovoltaicos e transformadores, lidando com os desafios de dimensionamento de projetos e simulação computacional e aprendendo também sobre comportamento de harmônicos e como analisá-los. Tudo visto de forma prática, pois puderam manipular e analisar parâmetros; barata, pois não foi necessário a compra de nenhum equipamento; à distância, pois toda comunicação entre os autores foi feita remotamente.

O aprendizado foi facilitado e reforçado por uma metodologia mais ativa e dinâmica, onde ao mesmo tempo em que os alunos buscam embasamento teórico devem analisar criticamente se o método encontrado é o melhor para solucionar o problema e o processo também é dinâmico, pois vê o efeito que cada parâmetro possui sobre o sistema através das análises gráficas feitas a partir da simulação. 
Portanto, esta metodologia de ensino se mostra eficaz, podendo também ser aplicada em outras áreas da engenharia e quaisquer instituições de ensino, especialmente às que possuem menos recursos de laboratório e sistemas EAD.

\section{Agradecimentos}

Agradecemos primeiramente à Deus por todas as bençãos. Agradecemos também às nossas famílias e amigos pelo apoio e ao CEFET/RJ por nos dar a oportunidade de estudo.

\section{REFERÊNCIAS}

CASARO, M. M.; MARTINS, D. C. Modelo De Arranjo Fotovoltaico Destinado A Análises Em Eletrônica De Potência Via Simulação, Eletrônica de Potência, v. 13, n. 3, p. 141-146, 2008.

CHAPMAN, Stephen J. Fundamentos de Máquinas Elétricas. $5^{\text {a }}$ edição, Porto Alegre: AMGH, 2013.

EPE. Anuário Estatístico de Energia Elétrica 2019 ano base 2018. Rio de Janeiro, 2019.

FREITAS, Duarte Nuno Santos. Controlo de um Inversor Monofásico para Ligação à Rede de um Gerador Síncrono de Ímanes Permanentes. 2013. 95 f. Dissertação (Mestrado), Engenharia Electrotécnica - Escola Superior de Tecnologia e Gestão, Instituto Politécnico de Bragança, Bragança, 2013.

MARTINS, Denizar Cruz; BARBI, Ivo. Eletrônica de Potência: Conversoress CC-CC Básicos Não Isolados. Florianópolis: Edição dos Autores. 2006.

MOJUMDAR, R. R.; BHUIYAN, W.; KADIR, H.; SHAKIL, N. H.; RAHMAN, A. U. Design $\&$ Analisys of na Optimized Grid-tied PV System: Perspective Bangladesh. IACSIT International Journal of Engineering and Technology, v. 3, n. 4, 2011.

PEREIRA, Filipe Miguel Costa. Conversor CC/CC elevador para painéis fotovoltaicos. 2012. 89 f. Dissertação (Mestrado) - Faculdade de Engenharia da Universidade do Porto, Universidade do Porto, Porto, 2012.

PEREIRA, O. L. S.; GONÇALVES, F. F. Dimensionamento de Inversores Para Sistemas Fotovoltaicos Conectados à Rede Elétrica: Estudo de Caso do Sistema de Tubarão - SC. Revista Brasileira de Energia, v. 14, n. 1, p. 25-45, 2008.

ROZENBLAT, L. A Grid Tie Inverter For Solar Systems. Disponível em: https://solar.smps.us/grid-tie-inverter-schematic.html. Acesso em: 13 abr. 2020.

SALDANHA, Emmanuel L. C.; MACHADO, Mateus C.; CARRARO, Cintia F. F.; FERNANDES, Camila B. Simulação e Análise de Potência de Placas Fotovoltaicas para Variações de Temperatura e Irradiação. In: XLVII Congresso Brasileiro de Educação em 
Engenharia e II Simpósio Internacional de Educação em Engenharia da ABENGE, 2019, Fortaleza. Anais. Fortaleza, 2019.

SILVA, Leonardo Rosenthal Caetano. Análise de Técnicas de Rastreamento de Máxima Potência (MPPT) para Aplicação em Arranjos de Sistemas Fotovoltaicos Conectados à Rede. 2015. 187 f. Dissertação (Mestrado), Engenharia Elétrica - Universidade Federal de Uberlândia, Uberlândia, 2015.

SOUSA, Karla Keitianne Ferreira de. Controle da Tensão do Barramento CC pelo Inversor Monofásico Ponte Completa em um Sistema Fotovoltaico Conectado à Rede Elétrica. 2016. 67 f. Monografia (Bacharelado) - Departamento de Engenharia Elétrica, Universidade Federal da Paraíba, João Pessoa, 2016.

TEIXEIRA, Douglas Ângelo. Análise das Distorções Harmônicas - Estudo de Caso de Sistema Industrial.2009. 19 f. Dissertação (Mestrado) - Universidade Federal de Minas Gerais, Belo Horizonte, 2009.

TIBA, C. et al. Atlas Solarimétrico do Brasil. Recife, 2000.

\title{
THE INFLUENCE OF SOLAR IRRADIATION ON HARMONICS GENERATED BY GRID-TIED SOLAR SYSTEMS AND ITS EFFECT ON THE FACTOR K OF THE SINGLE-PHASE TRANSFORMER
}

\begin{abstract}
With the increase in competitiveness in the job market, professionals need to accumulate more and more skills and knowledge in order to stand out in their careers. Thus, adopting a teaching method that develops skills and builds broad knowledge is challenging. Computer simulation is a skill of the future and combined with the observation of engineering problems and the multidisciplinarity of projects, it can be a good teaching method. Thus, in this work, the entire project of a photovoltaic system connected to the electrical network was made and it was sought to evaluate through computer simulation the effect of the harmonics generated by the system in a transformer. As a result, it was found that the levels of irradiation alter the levels of harmonics generated by the system, which can cause serious problems to the grid and to the transformer connecting to the grid by changing the $K$ factor of $i$. This teaching methodology that combines computational resources with the multidisciplinarity involved in engineering projects and the identification of trends in the electrical system proves to be an interesting model as it not only allows the development of skills but also the broad knowledge and critical eye of the engineer.
\end{abstract}

Keywords: Photovoltaic system. Grid-tied inverter. K factor. Transformer. Engineering challenge. 